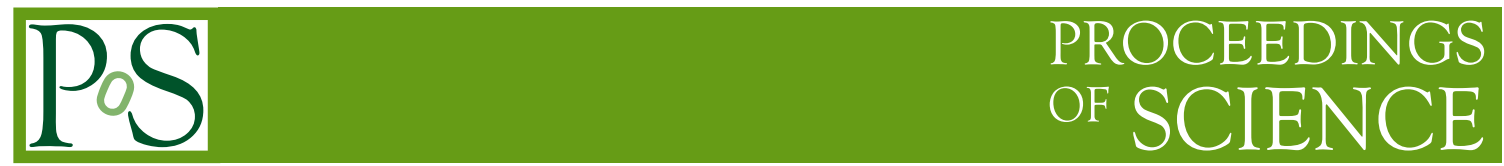

\title{
Status of the MUonE experimental proposal
}

\section{G. Venanzoni*i}

INFN Sezione di Pisa, Pisa, Italy

E-mail: graziano.venanzoniepi.infn.it

We present the status of the MUonE experimental proposal which aims at determining the leading order hadronic contribution to the muon $\mathrm{g}-2$ by measuring the hadronic part of the photon vacuum polarization in the space-like region.

European Physical Society Conference on High Energy Physics - EPS-HEP2019 -

10-17 July, 2019

Ghent, Belgium

* Speaker.

${ }^{\dagger}$ For the MUonE Collaboration 


\section{Introduction}

There is a tantalizing discrepancy of $\sim 3.5$ standard deviations between the measurement of the muon anomaly $a_{\mu}=(g-2) / 2$ performed by the E821 experiment at BNL and the Standard Model prediction [1]. Whether this discrepancy is real or not, it certainly calls for a more precise determination of $a_{\mu}$. New experiments at Fermilab (E989, an evolution of E821) and at J-PARC (E34, with a completely different technique) aim to measure $a_{\mu}$ to $0.14 \mathrm{ppm}$. With the planned improvement of the measurement, it is important that the theoretical prediction improves as well. The leading-order hadronic vacuum polarization contribution, $a_{\mu}^{\mathrm{HLO}}$, currently represents the main limitation for the theory due to the non-perturbative QCD behavior at low energy. An intense research program is underway with both time-like data and lattice calculations [2].

\section{The MUonE Project}

A novel approach has been proposed recently to determine the leading hadronic contribution to the muon $g-2\left(a_{\mu}^{\mathrm{HLO}}\right)$ measuring the effective electromagnetic coupling in the space-like region via scattering data [3]. The elastic scattering of high-energy muons on atomic electrons of a low- $Z$ target has been identified as an ideal process for this measurement, and a new experiment, MUonE, has been proposed at CERN to measure the shape of the differential cross section of $\mu e$ elastic scattering as a function of the space-like squared momentum transfer [4]. Assuming a $150 \mathrm{GeV}$ muon beam with an average intensity of $\sim 1.3 \times 10^{7}$ muons/s, presently available at the CERN muon M2 beamline, incident on a target consisting of 40 beryllium layers, each $1.5 \mathrm{~cm}$ thick, and three years of data taking, one can reach an integrated luminosity of about $1.5 \times 10^{7} \mathrm{nb}^{-1}$, which would correspond to a statistical error of $0.3 \%$ on the value of $a_{\mu}^{\mathrm{HLO}}$. The direct measurement of the effective electromagnetic coupling via $\mu e$ scattering would therefore provide an independent and competitive determination of $a_{\mu}^{\mathrm{HLO}}$. It would consolidate the muon $g$-2 prediction and allow a firmer interpretation of the upcoming measurements at Fermilab and J-PARC.

\subsection{The experiment}
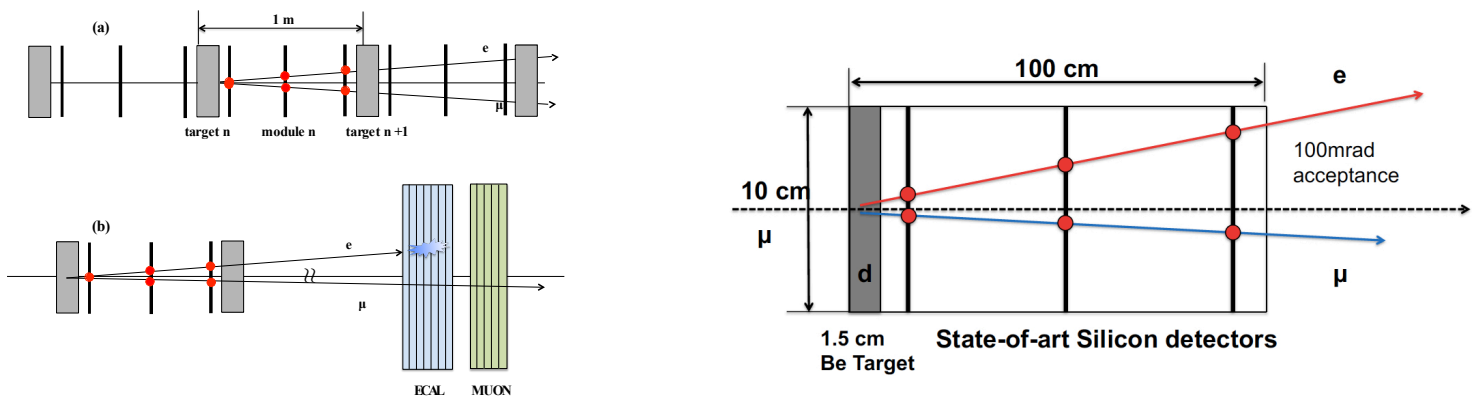

Figure 1: Left: design of the baseline detector concept. Right: single unit.

Figure 1 shows the baseline detector design. The detector is a repetition of 40 identical modules (called stations), each consisting of a $1.5 \mathrm{~cm}$ thick layer of Be coupled to 3 silicon tracking layers within a distance of $\sim 1 \mathrm{~m}$ (to be optimized) from each other with intermediate air gaps. 
Thin targets are required to minimize the impact of multiple scattering and the background on the measurement. Several targets allow to obtain the necessary statistics. The Si detectors provide the necessary resolution $(\sim 20 \mu \mathrm{m})$ with a limited material budget $\left(<0.06 X_{0}\right.$ per unit). This arrangement provides both a distributed target with low- $Z$ and the tracking system. Downstream of the apparatus a calorimeter and a muon system (a filter plus active planes) will be used for e/ $\mu$ particle identification. Significant contributions of the hadronic vacuum polarization to the $\mu e \rightarrow \mu e$ differential cross section are essentially restricted to electron scattering angles below $10 \mathrm{mrad}$, corresponding to electron energies above $10 \mathrm{GeV}$. The net effect of these contributions is to increase the cross section by a few per mille: a precise determination of $a_{\mu}^{\mathrm{HLO}}$ requires not only high statistics, but also a high systematic accuracy, as the final goal of the experiment is equivalent to a determination of the signal to normalization ratio with a $O(10 \mathrm{ppm})$ systematic uncertainty at the peak of the integrand function. Although this does not require knowledge of the absolute cross section (signal and normalization regions will be obtained by $\mu e$ data) it poses severe requirements on the knowledge of the following quantities:

- Multiple scattering: preliminary studies indicate that an accuracy of the order of $\sim 1 \%$ is required on the knowledge of the multiple scattering effects in the core region. Results from a Test Beam at CERN with electrons of 12 and $20 \mathrm{GeV}$ on 8-20 mm C target show good agreement between data and GEANT4 simulations, see Fig. 2 [5].
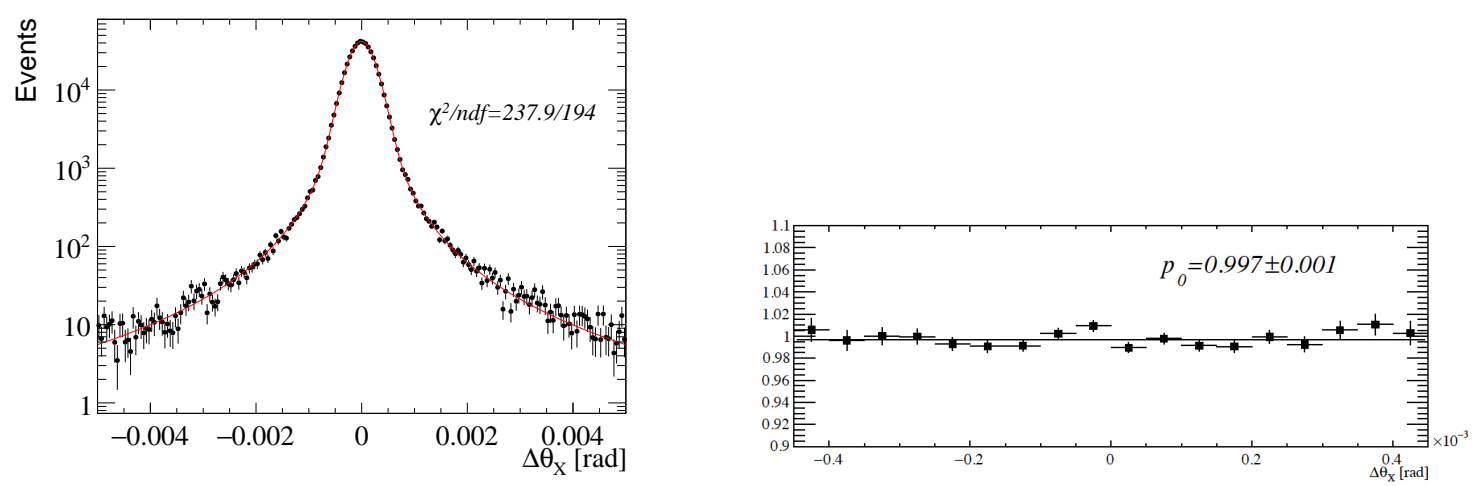

Figure 2: Left: x-projection (Left) of the scattering angle from $12 \mathrm{GeV} e^{-}$with $8 \mathrm{~mm}$ target compared with the results of the fit based on the sum of a Gaussian and a Student's $t$ distribution. Right: Data/Monte Carlo ratio.

- Tracking uniformity, alignment and reconstruction of angles: it is important to keep the systematic error arising from the non-uniformity of the tracking efficiency and angle reconstruction at the $10^{-5}$ level. The use of state-of-the-art silicon detectors should ensure the required uniformity. Among the considered alternatives, the silicon strip sensors being developed for the CMS Tracker upgrade represent a good solution. In particular, the silicon sensors which are foreseen for the CMS HL-LHC Outer Tracker (OT) in the so called 2S configuration have been chosen [6]. They are $320 \mu \mathrm{m}$ thick sensors with n-in-p polarity produced by Hamamatsu Photonics. They have an area of $10 \mathrm{~cm} \times 10 \mathrm{~cm}$ (sufficient to cover the MUonE acceptance) and a pitch $\mathrm{p}=90 \mu \mathrm{m}$, which means having a single hit precision 
$\sim p / \sqrt{12} \sim 26 \mu \mathrm{m}$. The strips are capacitively-coupled, and are segmented in two approximately $5 \mathrm{~cm}$ long strips. In the $2 \mathrm{~S}$ configuration two closely-spaced silicon sensors reading the same coordinate are mounted together and read out by common front-end ASIC. With their accompanying front end electronics they can sustain high readout rate $(40 \mathrm{MHz})$ and are well-suited for track triggering.
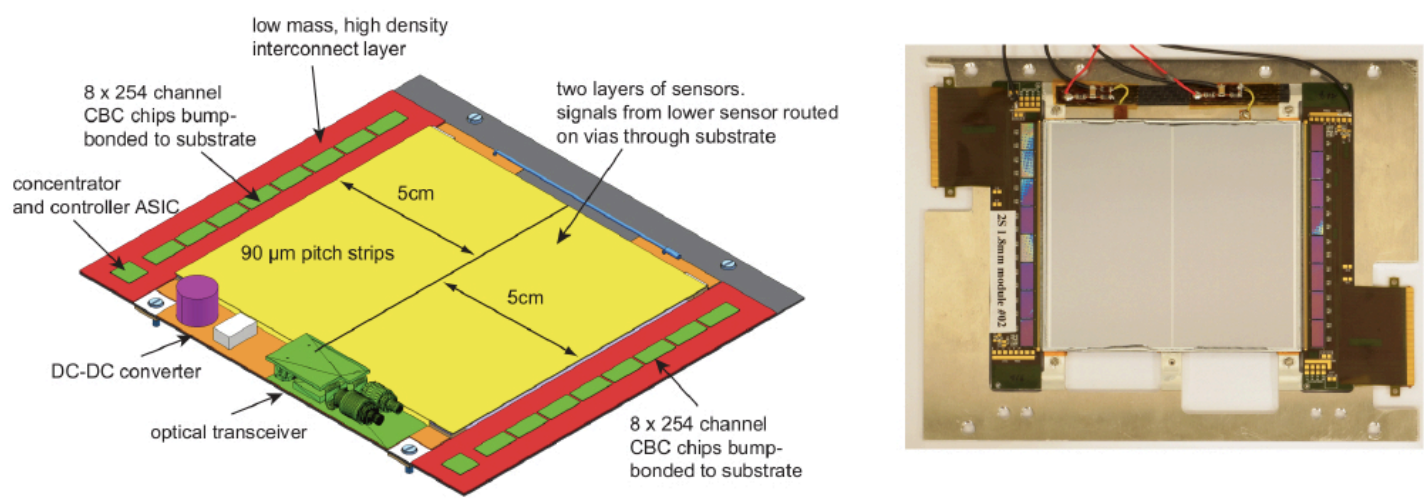

Figure 3: Left: A schematic view of the CMS 2S module. Right: A picture of the CMS 2S module.

The relative alignment of the silicon detectors will be monitored with the high statistics provided by the muon beam. The longitudinal position of the silicon detector must be controlled at the level of $10 \mu \mathrm{m}$. The support structure able to meet this requirement is shown in Fig. 4. The base is a $U$ shaped carbon fiber structure; the vertical dimension - $20 \mathrm{~mm}$ in figure guarantees stiffness to vertical sag. Carbon fiber has a negligible coefficient of thermal expansion (CTE) in the longitudinal direction, which can be theoretically reduced to zero in the region of interest $(\mathrm{T} \simeq 300 \mathrm{~K})$. Moreover its density $\mathrm{d}=1.42 \mathrm{~g} / \mathrm{cm}^{3}$ is very low, thus limiting the backscattering effect. The relative distance between the Si tracker elements will be monitored by a laser-interferometry system.

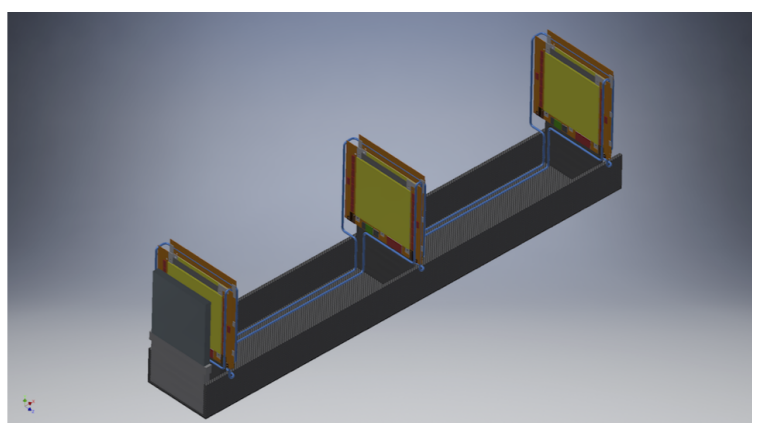

Figure 4: Support structure in carbon fiber.

- Knowledge of the beam: a $0.8 \%$ accuracy on the knowledge of the beam momentum, as obtained by the BMS spectrometer used by COMPASS, is sufficient to control the systematic 
effects arising from beam spread. The beam scale must be known at $\sim 5 \mathrm{MeV}$ level. This can be obtained by $\mu e$ elastic scattering events exploring the $\mu e$ kinematics [7].

- Extraction of $\Delta \alpha_{\text {had }}(t)$ in presence of NLO effects: The signal extraction is carried out by a template fit method. $\Delta \alpha_{\text {had }}(t)$ is modeled by a two-parameter analytical function with logarithmic dependency at large $|t|$ and linear behavior at small $|t|$, as expected from general principles [7]. Template distributions for the scattering angles $\theta_{\mu}$ and $\theta_{e}$, both 1D and 2D, have been calculated from NLO Monte Carlo events on a grid of points in the parameter space sampling the region around the expected reference values. The template fit is then carried out by a $\chi^{2}$ minimization, comparing the angular distribution of pseudodata with the predictions obtained for the scanned grid points. Extraction of $a_{\mu}^{H L O}$ is consistent with the expected value within half standard deviation.

\subsection{Theory}

The complete calculation of the full set of NLO QED corrections and of NLO electroweak corrections with the development of a fully exclusive Monte Carlo event generator for MUonE was completed in [8]. The generator is currently used for simulation of MUonE events in presence of QED radiation. The QED corrections at next-to-next-to-leading order (NNLO), crucial to interpret MUonE high-precision data, are not yet known. A first step towards the calculation of the full NNLO QED corrections to $\mu$ e scattering was taken in $[9,10]$, where the master integrals for the two-loop planar and non-planar four-point Feynman diagrams were computed. The NNLO hadronic corrections to $\mu$ e scattering have been computed very recently in $[11,12]$. A suitable subtraction scheme to deal with soft singularities at NNLO accuracy in QED, including finite fermion masses, has been presented in [13].

The extreme accuracy of MUonE demands for the resummation of classes of radiative corrections which are potentially enhanced by large logarithms. A general framework for implementing numerically the leading logarithmic resummations is provided either by the parton shower (PS) approach or the YFS formalism. These methods can be improved to include consistently NLO corrections $[14,15,16]$. Going one step further, when the complete NNLO corrections will be available and a NNLO matched PS (or $\mathscr{O}\left(\alpha^{2}\right)$ YFS) will be implemented, we expect that the error due to missing corrections will be $\mathscr{O}\left(10^{-6}\right)$ [7].

\subsection{Status and future plans}

At present, the MUonE Collaboration consists of groups from CERN, China, Germany, Italy, Poland, Russia, Switzerland, UK, and USA. These groups have strong expertises in the field of precision physics. A Letter of Intent has been submitted in June 2019 to CERNS SPSC [7]. The year 2020 will be devoted to continuing detector optimization studies, simulations, and theory improvement. The detector construction is expected during CERN LS2 and the plan is to have a first pilot run of a few weeks in 2021. A run at full statistics is envisaged in 2022-24. MUonE is part of the PBC Study Group at CERN [17]. 


\section{References}

[1] F. Jegerlehner, Springer Tracts Mod. Phys. 274 (2017); T. Blum, A. Denig, I. Logashenko, E. de Rafael, B. L. Roberts, T. Teubner and G. Venanzoni, arXiv:1311.2198 [hep-ph].

[2] Muon g-2 Theory Initative, see for example https://indico.fnal.gov/event/13795/.

[3] C. M. Carloni Calame, M. Passera, L. Trentadue and G. Venanzoni, Phys. Lett. B 746 (2015) 325.

[4] G. Abbiendi et al., Eur. Phys. J. C 77 (2017) no.3, 139.

[5] G. Abbiendi et al., arXiv:1905.11677 [physics.ins-det].

[6] CMS Collaboration. The Phase-2 Upgrade of the CMS Tracker. Tech. rep. CERNLHCC- 2017-009. CMS-TDR-014. Geneva: CERN, June 2017. https : / / cds . cern. ch/record/2272264.

[7] The MUonE Collaboration. Letter of Intent: The MUonE Project. CERN-SPSC-2019-026 / SPSC-I-252. 05/06/2019.

https://cds.cern.ch/record/2677471/files/SPSC-I-252.pdf .

[8] M. Alacevich, C. M. Carloni Calame, M. Chiesa, G. Montagna, O. Nicrosini and F. Piccinini, arXiv:1811.06743 [hep-ph].

[9] P. Mastrolia, M. Passera, A. Primo and U. Schubert, JHEP 1711 (2017) 198.

[10] S. Di Vita, S. Laporta, P. Mastrolia, A. Primo and U. Schubert, JHEP 1809 (2018) 016.

[11] M. Fael, JHEP 1902 (2019) 027.

[12] M. Fael and M. Passera, Phys. Rev. Lett. 122 (2019) no.19, 192001.

[13] T. Engel, A. Signer and Y. Ulrich, arXiv:1909.10244 [hep-ph].

[14] G. Montagna, O. Nicrosini and F. Piccinini, Phys. Lett. B 385 (1996) 348.

[15] G. Balossini, C. M. Carloni Calame, G. Montagna, O. Nicrosini and F. Piccinini, Nucl. Phys. B 758 (2006) 227.

[16] S. Jadach, W. Placzek and B. F. L. Ward, Phys. Lett. B 390 (1997) 298.

[17] R. Alemany et al., arXiv:1902.00260 [hep-ex]; A. Dainese et al. [QCD Working Group], arXiv:1901.04482 [hep-ex]. 